\title{
IMAGE PROCESSING TECHNIQUE TO COUNT THE NUMBER OF LOGS IN A TIMBER TRUCK
}

\author{
Asif Rahman ${ }^{1,2}$, Siril Yella ${ }^{1}$, Mark Dougherty ${ }^{1}$ \\ ${ }^{1}$ Department of Computer Engineering, Dalarna University, Borlänge, Sweden \\ ${ }^{2}$ Department of Information Technology, Örebro University, Örebro, Sweden \\ aus@du.se, sye@du.se, mdo@du.se
}

\begin{abstract}
This paper summarises the results of using image processing technique to get information about the load of timber trucks before their arrival using digital images or geo tagged images. Once the images are captured and sent to sawmill by drivers from forest, we can predict their arrival time using geo tagged coordinates, count the number of (timber) logs piled up in a truck, identify their type and calculate their diameter. With this information we can schedule and prioritise the inflow and unloading of trucks in the light of production schedules and raw material stocks available at the sawmill yard. It is important to keep all the actors in a supply chain integrated coordinated, so that optimal working routines can be reached in the sawmill yard.
\end{abstract}

\section{KEY WORDS}

Timber Trucks, Logistics, Supply chain, Sawmill, Image processing and Geo tagging.

\section{Introduction}

In this current work the sawmill yard operations of Bergkvist Insjön $\mathrm{AB}^{I}$ are examined. The Bergkvist Insjön (sawmill) is facing several internal problems due to unexpected arrival of timber trucks, which can't be controlled by them. Despite being equipped with modern technology for handling operations, it is still facing several challenges threatening the overall efficiency and thereby limiting the profit margin of sawmill. The sawmill yard operations starts with unloading of logs from truck on to measurement buffer, from the measuring buffer to storage bins by assigning classes to each log according to its length and diameter, emptying the storage bins and piling up the logs in storage area and finally feeding the sawmill with $\operatorname{logs}$ for production. Given the huge production volume, it is obvious that the efficient handling of resources is a top priority. A particular challenge in efficient handling concerns with the unpredictable arrival of trucks loaded with timber, usually in the morning hours. The trucks loaded with timber arrive at the sawmill to get unloaded; they can be emptied either onto the measurement buffer or onto the ground before they can leave sawmill yard. Unloading on the ground will cost more than double in handling because once unloaded on the ground the logs must be lifted again to be placed on the buffer whenever there is spare capacity available. The unloading of trucks has to be prioritised, trucks which are more important with respect to the type timber they carry are unloaded on buffer and remaining trucks are either unloaded on the ground or have wait in a queue. The resources available to unload the raw material can only unload 5-6 truck an hour, further sawmill owners has to pay a waiting fee for drivers if it exceeds 30 minutes in a queue. The Sawmill cannot predict the arrivals of trucks so at times there will be $20-25$ trucks in a queue; other times there will be only 5 to 6 in the queue.

To predict the arrival of external logistics (timber trucks) the geo tagging of images has been proposed, the data embedded in the Geo tagged images have associated GPS coordinates and a timestamp [1]. Such geo-tagged images provide us with information of what, when and where the truck drivers are before they depart for the sawmill (Bergkvist Insjön). The image itself is a photograph of the rear end of the truck trailer. Once the images have been received, their geo coordinates (latitude and longitude) are extracted and a distance is calculated to predict their arrival time to destination. Further, the image can be used to identify what the truck is carrying (type of $\log$ ); how much they are carrying and what are the diameters of those logs. This information is important aspect for scheduling and prioritizing the in-use log stackers (resources) in sawmill yard operations. This paper is limited only to deducing information regarding the truck load i.e. to know how many logs they are carrying. Several image analysis techniques were applied for each image to segment the logs within the image and to derive the required information. Sample images were tested with our algorithm and we achieve an average of $97 \%$ success in counting the number of logs in a truck from an image.

The rest of the paper is organized as follows; Section 2 provides an introduction to brief experimental setup and design. Then, Section 3 explains about methodology development. Section 4, describes some results and analysis and finally, Section 5 presents conclusion and future work.

${ }^{1}$ http://www.bergkvist-insjon.se/860.php 


\section{Experimental Setup and Design}

Image processing techniques were considered appropriate. The idea is to segment all the logs which are piled up in the rear end of a truck, and generate the results concerning number of $\operatorname{logs}$ in it. The algorithm has to process the static image and segment the object of interest individually and it has to be capable of discriminating between noise and other undesired material, mainly composed of trailer, poles, wires etc. (see Figure 1).

Segmentation consists of determining which regions of the image correspond to background and which represent the objects of interest [2]. The pixel-oriented segmentation algorithm has been used, because these algorithms are normally faster than other approaches. From these algorithms the required object of interest from the whole image can be displayed. Once the background had been removed, each connected region was labelled as a possible object of interest. In the same operation, the program separates each log from whole pile using a superimposed image of log edges; further small objects were removed, individual objects were counted, numbers were assigned, the image log radius is calculated and a circle is drawn around it to make sure the object fell into it.

The unwanted material such as noise is removed by pre-processing of each image. Finally, the objects (logs) are segmented without its background on the new screen. The optimal solution had to be the one that consumed less computing time and was easier to operate by a nonexperienced user, without compromising quality standards.

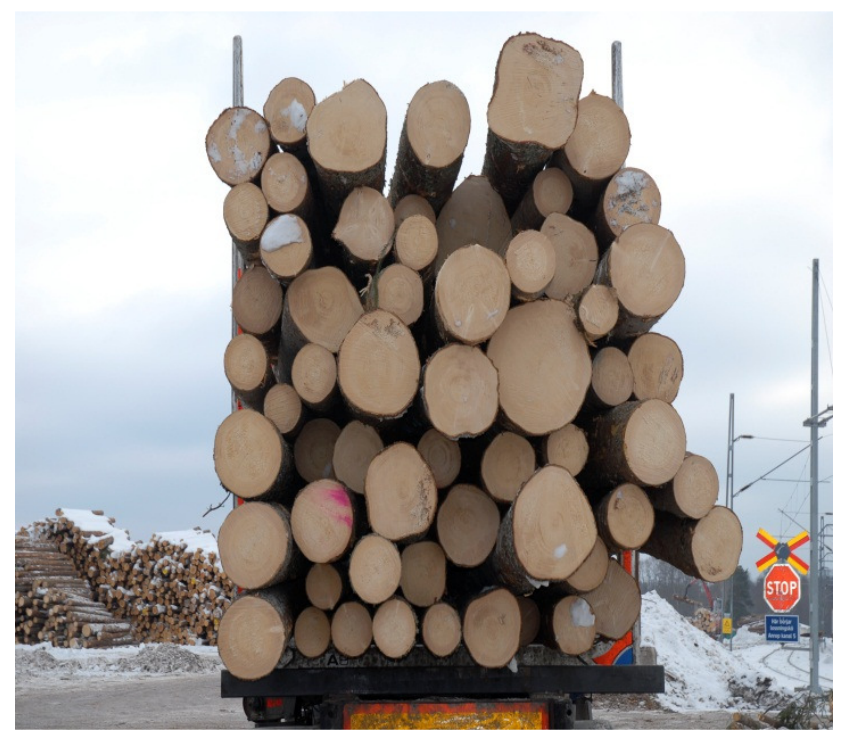

Figure 1. Spruce Timber Logs

The first stage of any automatic vision system is the image acquisition phase. The image acquisition plays major part in this work, as the data has to be acquired very clearly so that segmentation becomes very easy. It depends up on how good the camera calibration is.

Camera calibration is used to get information of the real world or we can say as primarily, finding the quantities internal to the camera that affect imaging process. All the images in this experiment were acquired using a same digital camera (Nikon D200) with approximately same distance from the truck. Two different set of images have been acquired, one belong to spruce and other belong to pine, as these are the requirement of Bergkvist Insjön and only these types of logs will arrive at the sawmill. (see Figures 1 and 3)

Before proceeding any further it is worth mentioning is that, only the timber truck image from rear end of a trailer has been considered in this work. The rear end of a truck is considered to be the key while determining the number of logs available in each truck trailer, the rear end of a pile image is segmented and number of logs was determined. Further the number is multiplied by 3 as each truck has 3 piles in its trailer, and overall sum has been considered to be average number of logs in a truck. A typical timber truck trailer contains three carriages and it is quite difficult to take individual carriage images, so we proceeded with the rear carriage. This relies on the assumption that a given truck has a homogenous load; in general this assumption is reasonable since the timber harvested from a particular forest site tends to be reasonably uniform in quality. The different set of images for both the types has been tested to check the accuracy of algorithm; however, it is worth mentioning that this experiment is a proof of concept to demonstrate the possible solutions to optimise operational routines.

\section{Methodology}

The design in this methodology starts with the image acquisition process, where a raw data is acquired through camera and send to the processing unit. The (Figure 2) can be seen as a design of real time object identification process based on series of computer vision technologies [3]. The main objective here is to detect the object and represent it in a better way. In the detection step, acquired images are pre-processed, enhanced and segmented according to object properties such as colour and shape. Only the regions of interest are extracted from the complex background and represent it in another mask. The time taken to respond the data will be more if it takes more time in pre-processing. Meanwhile, the segmented object is imposed against canny edges of original image; further connected component labelling is done to identify individual objects. Once the individual objects are segmented and each object calculates its length and width and further radius is calculated to draw a circle around it. All the selected objects are numbered to count number of 
logs in it and sum up with the three piles or carriages and generate the result.

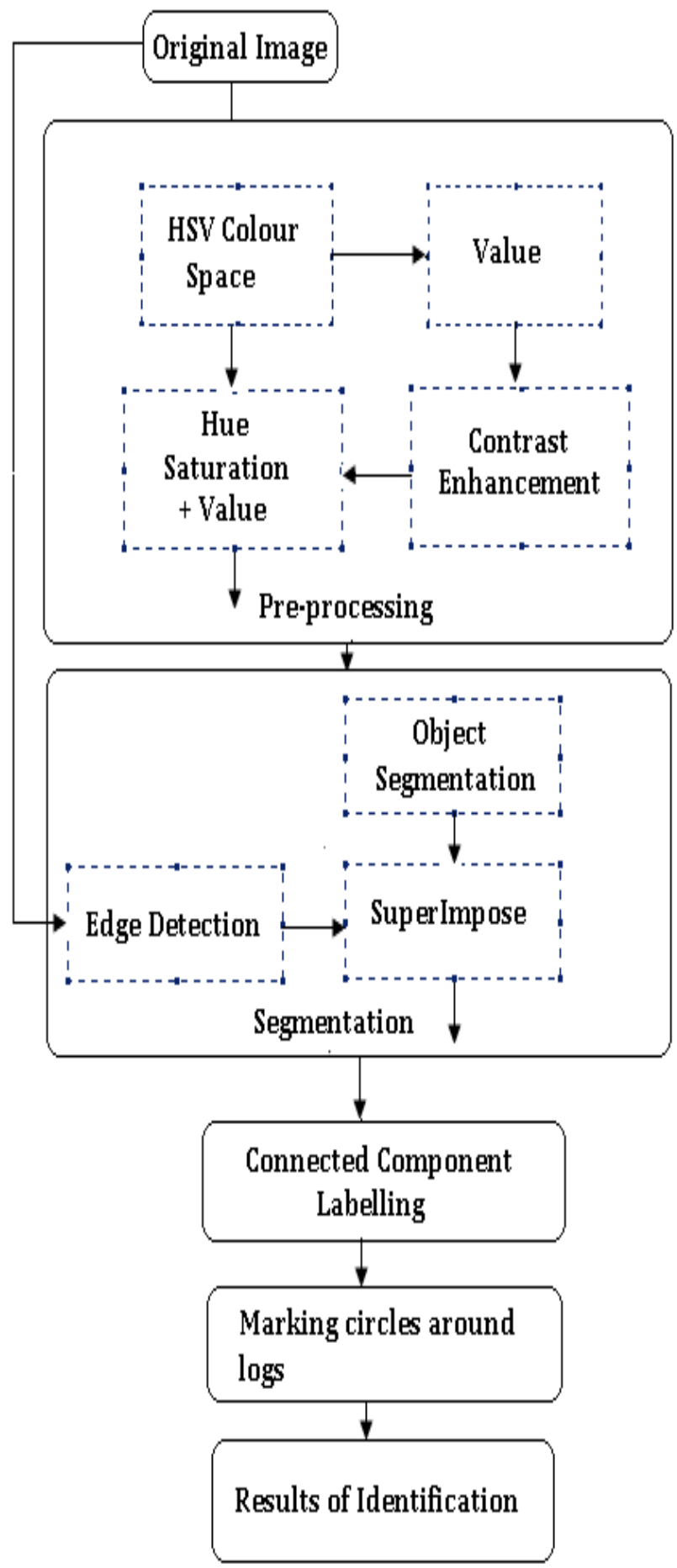

Figure 2. Flow Chart

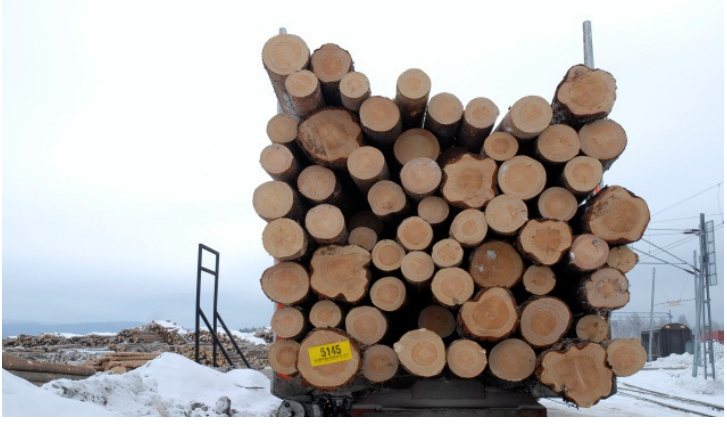

Figure 3. Pine Timber Logs

\subsection{Pre processing}

The Opencv library will accept almost all the formats; however, the format can be changed depending upon requirement. The images acquired are in RGB colour format and their dimensions are resized to $600 \times 600$ pixels, for easy processing without any loss in resolution. But, before segmenting pre-process techniques have been used because whenever image are acquired by a camera, often the vision system for which it is intended is unable to use it directly in segmentation. Random variations in intensity, variations in illumination or poor contrast could corrupt the data. This must be dealt with in early stages of vision processing [4]. In this experiment real time images of truck were acquired and processed to get the desired results. In the first step, the original image was converted into to two different forms, the first one was to apply canny edge detection operator to identify all the necessary edges of timber logs and other was to work on segmentation part. First the canny edges we determined by using the canny operator from opencv with parameters of 30,160 (threshold values) and apparatus size 3 has been used; further the dilation operator has been used with single iteration to have a thick boundary between individual logs. Second on was to segment the Image as timber logs exhibits certain colour and it will be easy to segment that colour.

The space of a color image shown in the computer is commonly the RGB color space. One of the big disadvantages of using the RGB colour space is its difficulty to separate the colour information from the brightness one. The three components of a color image have strong correlation and the color will distort or shift when any component of a pixel is changed. Instead of using the colour primaries, the HSV uses colour descriptions which have a more intuitive appeal to users. To give a colour specification, a user selects a spectral colour and the amounts of white or black which is to be added to obtain different shades, tints, and tones [4]. 
The pre-processing depends upon the data; if the data is noisy pre-processing techniques are necessary before converting to HSV. The data acquired for this experiment didn't require any pre-processing technique before converting to HSV. However, pre-processing has been done after converting to HSV by enhancing the value channel. The objective of image enhancement is to process an image to enhance its contrast or brightness depending up on the requirement what users want to perceive from image. Further, colour image enhancement can be classified into two categories according to colour space. The RGB image enhancement based on Histogram Equalization, Specification to the R, G, and B channels results in colour distortion according to [2], due to strong correlation and the ratio of three components. The second approach of colour enhancement is based on transform space, where RGB image is converted to HSV and process the value component and saturation component separately; however, we concentrated only on value channel. The contrast enhancement was done by (Look Up Table) function from opencv with delta value of 82 has been selected after few sample tests with less contrast and more contrast images. With increase in value channel the objects were visible more clearly and can be segmented as well, further the value channel image were merged with the other channel images and converted back to RGB space for segmentation purposes.

\subsection{Image Segmentation}

This technique is used to identify the object of interest in image. Or its purpose is to identify the object and discard remaining pixels of image. After image acquisition each image is pre-processed to clean the noise and to get more details form it. The RGB image was separate into three channels and we enhances the intensity or value channel as mention above, after enhancement we combine the three channels and converted back to RGB image. The enhanced RGB image was normalised by splitting the channels and dividing each pixel value by the sum of pixel's value over all channels gives you the normalised channel, and merging them back gives you the normalised RGB image. Further, the normalised image was converted to HSV for the segmentation purposes. From the HSV planes, the HSV values of the each pixel are normalized be in range of (0 and 1$)$.

In the current case it has been experimentally observed that color of logs possesses a hue value that is in between 0.05 and 0.15 ranges. So for segmentation of the logs, all the pixels within this range are kept and the rest of the pixels are discarded thereby leaving the frame mainly with the logs on a binary image (see Figure 6(e)). More recently good level of detail concerning color differences in the HSV color space has been detailed in [5].

Once the object of interest has been segmented and displayed in the other mask, the canny edges of original image are superimposed against the segmented one to create a geometric projection of logs on segmented image (see Figure 6(f)). The edges will act as boundary between individual objects $(\operatorname{logs})$, once the boundary (edges) has been added to the segmented image, the boundary pixels values are merged with the background of segmented image, leaving only the individual logs separated from each other. (see Figure $(6(\mathrm{~g}))$

\subsection{Counting the Number of Logs}

Further, connected component labelling has been applied with an aim to identify all the objects in the image. A connected component labelling algorithm finds all connected components and assigns a unique level to all points in the same component [3]. Labelling could be done either 4 or 8 , where 4 specify 4 connected objects and 8 specifies 8 connected objects where diagonal connections are possible. However, in this work the contour method from opencv has been used, where the function retrieves contours from the binary image and returns the number of retrieved contours. The area of each contours has been calculated and the area less than 300 pixels has been deleted to avoid the background noise

In this stage of processing, the algorithm gets the bounding rectangle of all contours and calculates its length and width, once the length and width are known, hypotenuse has been considered to know the diameter of the log. Further, the length, width and diameter have been divided by 2 to get the centre point of the log and the radius of the log. Once the radius (image) has been calculated a circle is drawn according the radius from centre of contour or object. However, the radius calculated will be more than actual radius because the contours act as bounding rectangle. In this way all the log objects are counted, numbered and circles are drawn around it. For better understanding step by step procedure is mention in the (Figure 5 and 6).

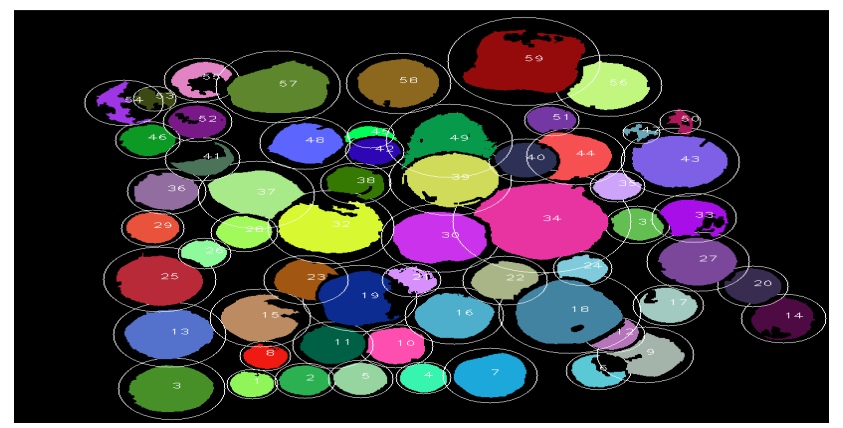

Figure 4: Marking Circles around each log 


\section{Results and Discussion}

The work in this article has made an experimental attempt to count the number of logs in a timber truck using images from rear end of a trailer. The segmentation results shows that, some images segmented more than actual logs and some images segmented less than actual logs. The average $\log$ segmentation gives around $97 \%$, where individual log segmentation varies between $92 \%$ and $102 \%$. However, variation in the results of individual logs are didn't matter much as the aim of this experiment is to calculate the average number of $\operatorname{logs}$ in a truck. The increase or decrease of few logs doesn't make any difference as results are very much closer to actual average.

Table 1

Result of using Image processing techniques to count the number of logs.

\begin{tabular}{|c|c|c|c|}
\hline Images & Actual & Segmented & Type \\
\hline 1 & 58 & 59 & Spruce \\
\hline 2 & 45 & 44 & Spruce \\
\hline 3 & 78 & 73 & Spruce \\
\hline 4 & 53 & 48 & Spruce \\
\hline 5 & 65 & 61 & Spruce \\
\hline 6 & 60 & 61 & Spruce \\
\hline 7 & 53 & 54 & Pine \\
\hline 8 & 90 & 85 & Spruce \\
\hline 9 & 62 & 63 & Pine \\
\hline 10 & 69 & 70 & Pine \\
\hline
\end{tabular}

The results mention in table 1 are from both types of timber images Spruce and Pine, from the computational point of view, this experiment didn't take much time to perform all the required operations. For each image it takes around 2-3 seconds to display all the resulted images and generate the results. However, the result may not be statistically significant to validate the experiment nevertheless the experiment was a proof of concept to demonstrate the possible solution to optimise the working routines in Sawmill yard. Few results suggested that there might be false positives and false negatives, as logs in the image are very close to each other. However, the addition or subtraction of few logs here and there wouldn't matter that much. As main goal is count the average number of logs.

\section{Conclusion and Future work}

A promising approach for segmenting and counting number of log has been presented. To keep the internal resources of Bergkvist Insjön optimised. The drivers have to capture the images and send it to Bergkvist Insjön before their arrival. With the image we predict their arrival time, identify the type they are carrying and count how many logs they are carrying with in few seconds. In this work $\log$ extraction with normal images has been considered, working with geo tagged images, identifying the type of log, calculating original diameter from image and giving feedback to drives about possible serving time has been left for future work.

The images from rear end of a truck have been used in the current case. Appropriate image based processing has been carried out, to be able to detect individual logs without any noise. Results achieved in this current work are around $97 \%$, by taking average percentage of segmented results, however, we achieve this result provided that image contrast and intensity are set to appropriate levels. Work done in this paper is a part of another major project where every information is sent to a simulation used to develop optimal decision, if we are dealing with $\mathrm{x}$ number of logs within few hours.

This work can be extended further by extracting individual logs from the circles drawn and identifying their types by identifying the pith and ring [6]. Further, geo tagged coordinates will be extracted and traveling distance is calculated. However, overall aim is give as much as possible information about arrival of timber trucks to Bergkvist Insjön before their arrival.

\section{REFERENCES}

[1] Jiannong Xin, "Tracking Pests and Plant Diseases Using Geo Tagged Digital Images", 2009

[2] Song G, Qiao X, "Adaptive Color Image Enhancement Based on Human Visual Properties", China, 2008

[3] Ramesh J, Rangachar K, Schunck B G, "Machine Vision", 1995.

[4] Gonzalez R. C., Woods R. E., "Digital Image Processing Handbook", Kluwer, 1998

[5] C. Millet I. Bloch and A. Popescu"Using the Knowlege of Object Colors to Segment Color and Improve Web Image Search" France, 2007

[6] Kristin N, Gunilla B, "Estimation of Ptih position is untreated log ends in sawmill environment", Sweden 2008 
Timner Truck (Pine)

a)

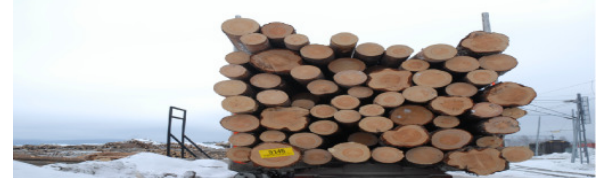

b)

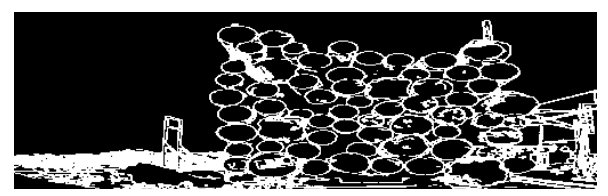

c)

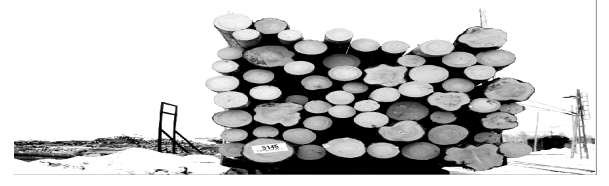

d)

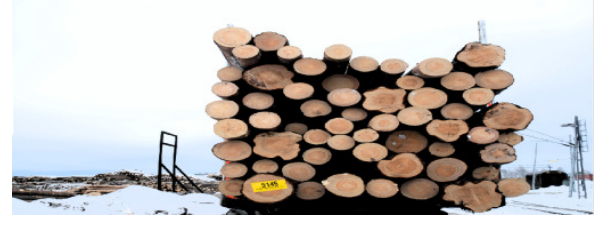

e)

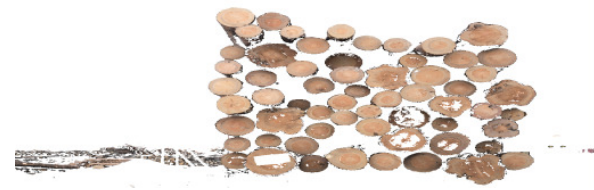

f)

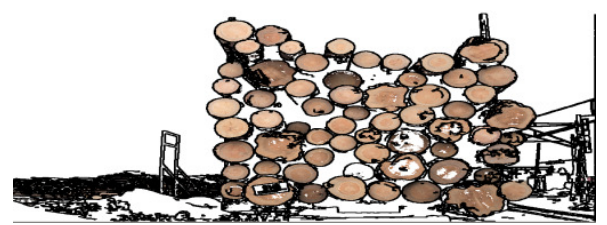

g)

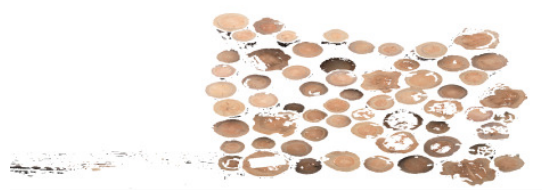

h)

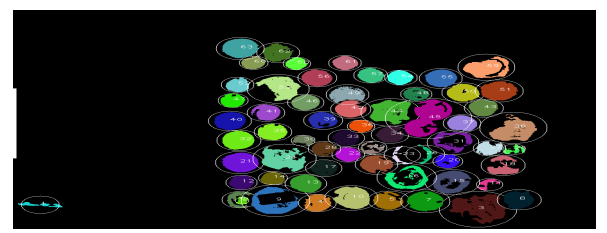

Figure 5. Various stages of processing

(a) Original Image (b) Canny Edge (c) Enhancement of Value channel (d) Enhanced RGB Image (e) Results of segmented Image (f) Super Imposed with canny edges (g) Separation of individual objects (h) Counting number of logs in a truck and marking circles around it. a)

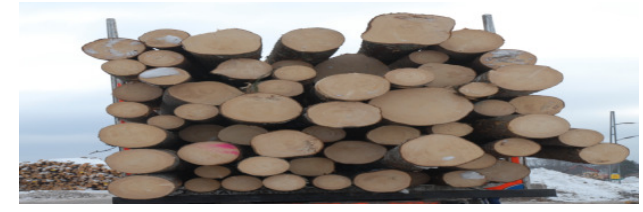

b)

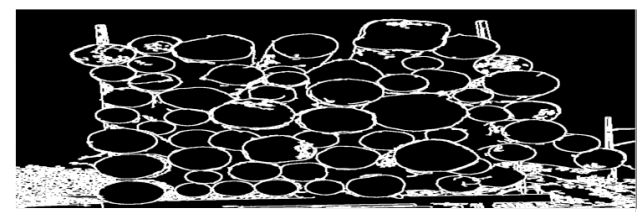

c)

d)

e)

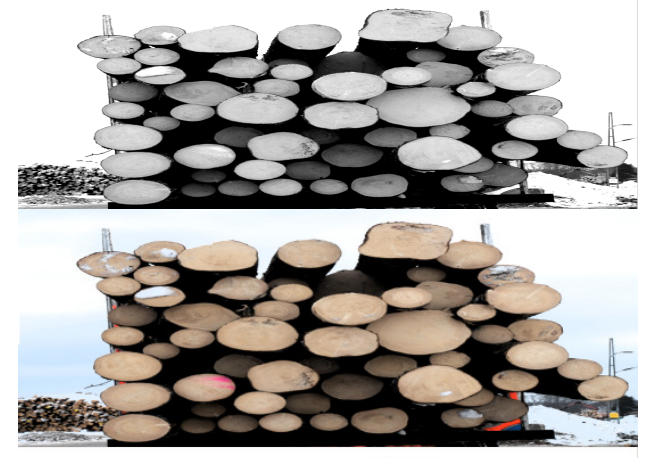

f)

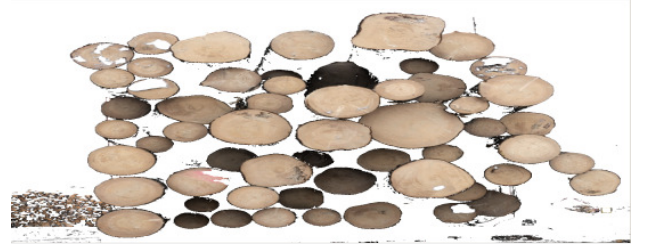

g)

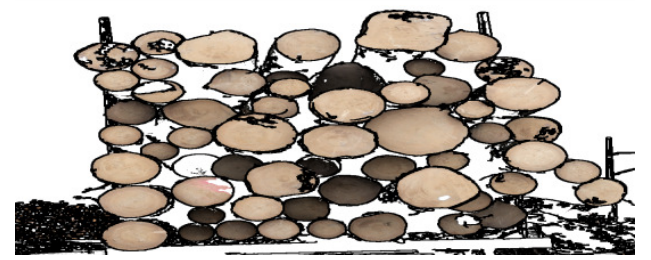

h)

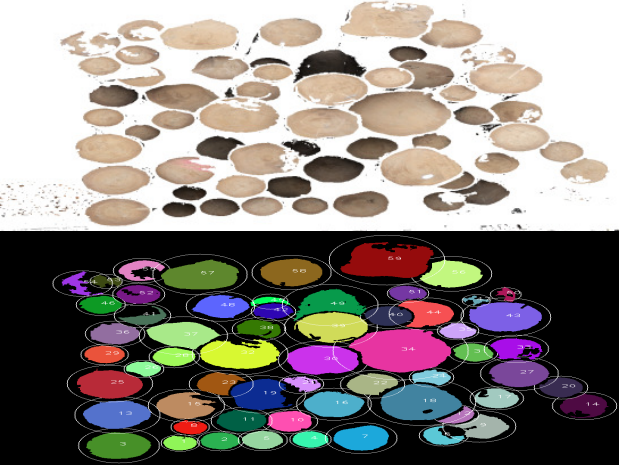

Figure 6. Various stages of processing

(a)Original Image (b) Canny Edge (c) Enhancement of Value channel (d) Enhanced RGB Image (e) Results of segmented Image (f) Super Imposed with Canny edges detection (g) Separation of individual objects (h) Counting number of logs in a truck and marking circles around it. 
a)

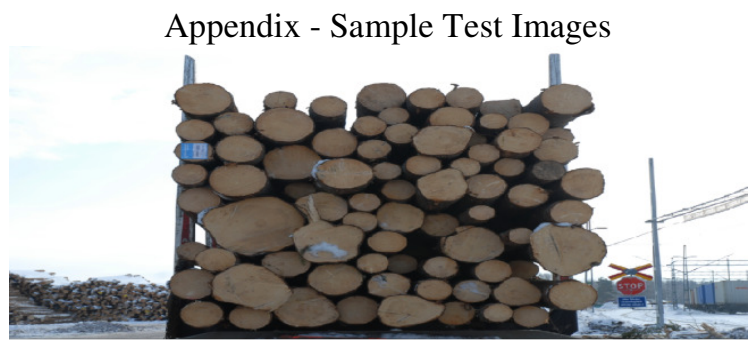

b)

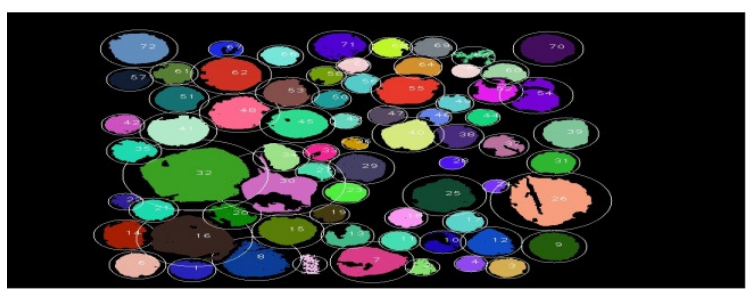

c)

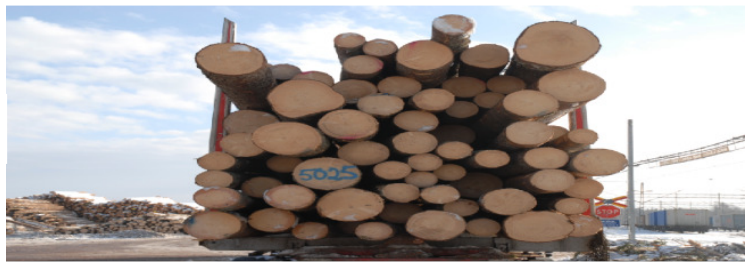

e)

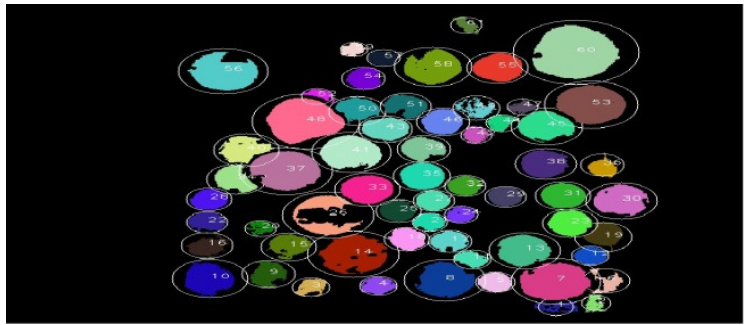

e)

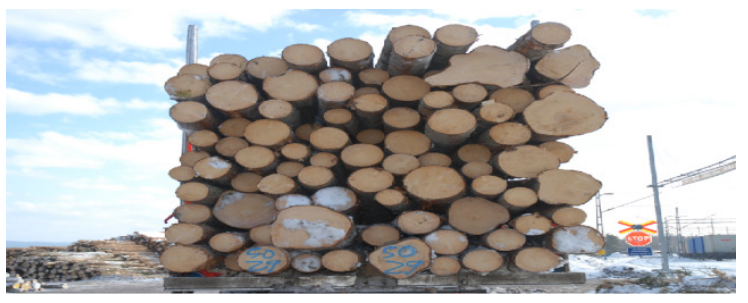

f)

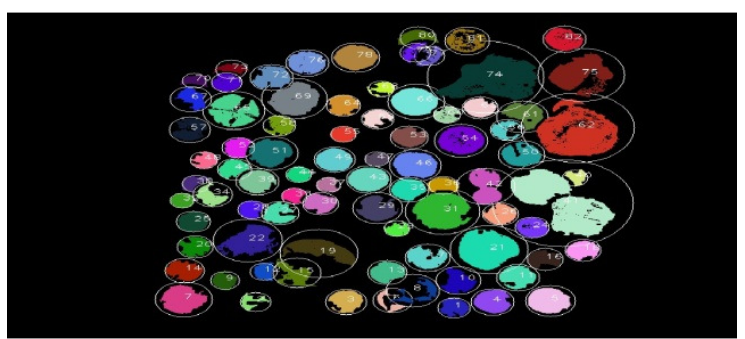

Figure 7 Sample Test Images
(a) (c) (e) Original Image (b) (d) (e) Counting logs and marking circles around it 